\title{
Prosthodontic Management of Congenital Hypothyroidism with Anodontia: A Case Report
}

\author{
Varsha Bhat ${ }^{1}$, Vidya S Bhat ${ }^{2}$, Jamsheera Vadakkan ${ }^{3}$, Sham S Bhat ${ }^{4}$, Sanath Shetty ${ }^{5}$, Sundeep K Hegde ${ }^{6}$
}

\begin{abstract}
Thyroid dysfunction has various manifestations which include orofacial abnormalities like delayed eruption, retained deciduous teeth, etc. Early detection of this deregulation of thyroid homeostasis can prevent associated complications. This report is a case of congenital hypothyroidism $(\mathrm{CH})$ in a 4-year-old boy who presented with a completely edentulous maxilla and hypodontic mandible. Based on various biochemical and radiographic investigations, a diagnosis of $\mathrm{CH}$ was established. He was prosthodontically rehabilitated with removable dentures.

Keywords: Anodontia, Congenital hypothyroidism, Pediatric prosthodontics.

International Journal of Clinical Pediatric Dentistry (2021): 10.5005/jp-journals-10005-1994
\end{abstract}

\section{INTRODUCTION}

Congenital hypothyroidism $(\mathrm{CH})$ is defined as thyroid hormone deficiency present at birth. ${ }^{1}$ It occurs when the thyroid gland fails to develop or function properly. Congenital hypothyroidism may be primary, due to a defect affecting the thyroid gland itself, or central, due to impaired thyroid-stimulating hormone (TSH)-mediated stimulation of the thyroid gland as a result of hypothalamic or pituitary pathology. There are five primary types of $\mathrm{CH}$ : thyroid dysgenesis, thyroid dyshormonogenesis, TSH receptor insensitivity, central $\mathrm{CH}$, and transient $\mathrm{CH}$.

The thyroid gland is located in the front portion of the neck below the larynx. It produces two hormones: triiodothyronine (T3) and thyroxine (T4), whose function is to control metabolism. ${ }^{2}$

Thyroid hormones play a very crucial role in the physical and mental systems in fetal and postnatal life. If iodine deficiency occurs very early during pregnancy, infants may present with severe growth failure, coarse facial features, intellectual disability, and spasticity.

In infants born with $\mathrm{CH}$, clinical features may not be obvious for several weeks or may display mild effects that often go unrecognized. Symptoms may be subtle or develop slowly because some maternal thyroid hormone crosses the placenta. As there are no conspicuous signs or symptoms, the diagnosis of $\mathrm{CH}$ is established only by a screening of the newborn.

Congenital hypothyroidism is characterized by increased sleep, feeding difficulty, constipation, prolonged jaundice, and neurocognitive decline. Also, newborns with $\mathrm{CH}$ show myxedematous facies, large fontanels, macroglossia, a distended abdomen with umbilical hernia, and hypotonia. ${ }^{3,4}$

Common oral features in $\mathrm{CH}$ include thick lips, a large tongue (macroglossia), delayed eruption of teeth, malocclusion, poor periodontal health, altered tooth morphology, and delayed wound healing. ${ }^{5-7}$ Neonatal screening (NS) for $\mathrm{CH}$ is not performed in all countries. Early detection helps in better treatment planning in case of missing teeth. Prosthetic rehabilitation plays a vital role in esthetics, mastication, and the growth and development of the jaws.

The present case report is of early dental prosthetic rehabilitation in a male child affected with $\mathrm{CH}$.
1,2,5 Department of Prosthodontics, Yenepoya Dental College, Yenepoya
University (Deemed to be University), Mangaluru, Karnataka, India
${ }^{3}$ Department of Pedodontics, Yenepoya Dental College, Yenepoya
University (Deemed to be University), Mangaluru, Karnataka, India
4,6 Department of Pedodontics and Preventive Dentistry, Yenepoya
Dental College, Yenepoya University (Deemed to be University),
Mangaluru, Karnataka, India

Corresponding Author: Varsha Bhat, Department of Prosthodontics, Yenepoya Dental College, Yenepoya University (Deemed to be University), Mangaluru, Karnataka, India, Phone: +91 8123568932, e-mail: varshapbhat3@gmail.com

How to cite this article: Bhat V, Bhat VS, Vadakkan J, et al. Prosthodontic Management of Congenital Hypothyroidism with Anodontia: A Case Report. Int J Clin Pediatr Dent 2021;14(4):586-589.

Source of support: Nil

Conflict of interest: None

\section{Case Description}

A 4-year-old boy was referred to the Department of Pedodontics and Preventive Dentistry, Yenepoya Dental College, Mangaluru, Karnataka, India, with the absence of teeth as a chief complaint. Parents became aware of his condition on immediate screening of blood after his birth in a Gulf country. Diagnosis of $\mathrm{CH}$ was confirmed by the pediatrician. The child has been on oral supplements of thyroxin ever since. Family history revealed the patient was the youngest child among the three siblings. He was born at full term, in a normal delivery. Mother is diagnosed with hypothyroidism and is on medications. His two elder sisters were normal. He was reported to have normal milestones. Parents had consulted a dentist when the child was 2 years old with the complaint of non-eruption of teeth, but no treatment could be done as he was too young to co-operate.

On extraoral examination (Fig. 1), patient appeared healthy, well-nourished with normal physical activity. Skin was normal and lips were unsupported. Face showed dished in appearance of the lower half. Speech was slightly slurred and the child was very un-cooperative.

(c) The Author(s). 2021 Open Access This article is distributed under the terms of the Creative Commons Attribution 4.0 International License (https://creativecommons. org/licenses/by-nc/4.0/), which permits unrestricted use, distribution, and non-commercial reproduction in any medium, provided you give appropriate credit to the original author(s) and the source, provide a link to the Creative Commons license, and indicate if changes were made. The Creative Commons Public Domain Dedication waiver (http://creativecommons.org/publicdomain/zero/1.0/) applies to the data made available in this article, unless otherwise stated. 


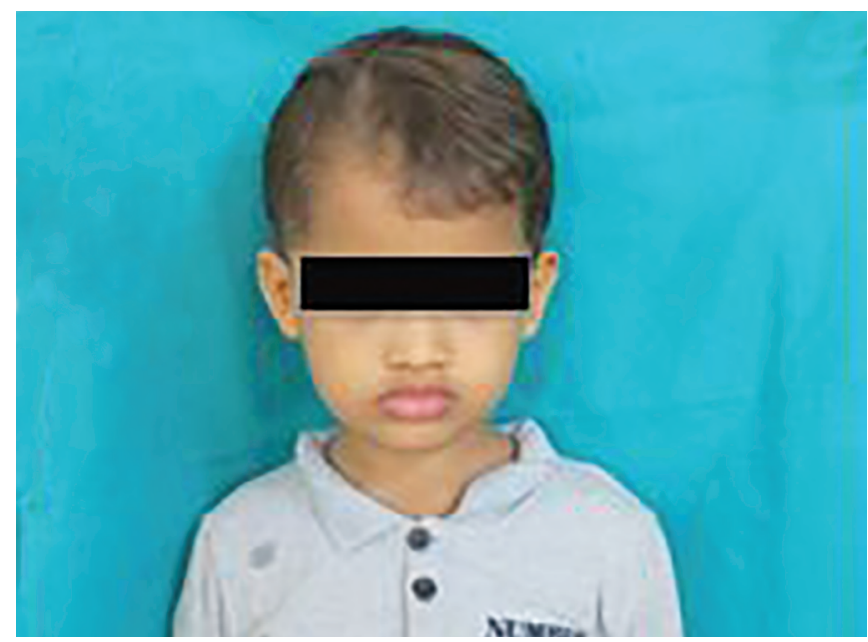

Fig. 1: Extraoral view—front profile (before treatment)

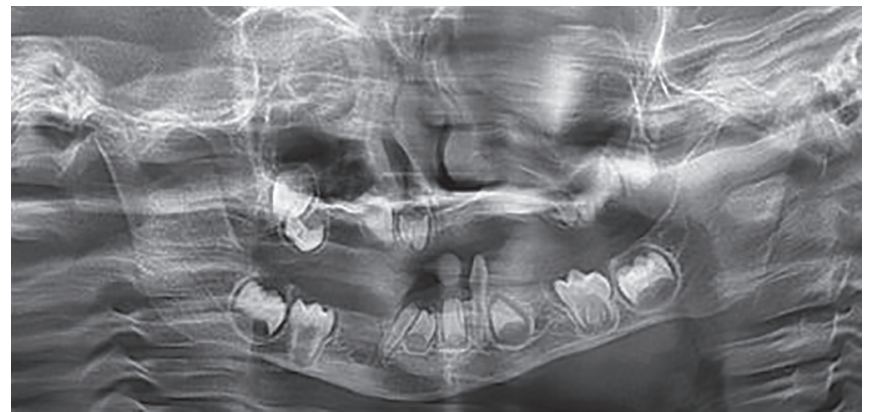

Fig. 3: Panoramic radiographic image

Intraoral examination showed a completely edentulous maxillary arch, oligodontia in the mandibular arch with only one deciduous mandibular canine (73) clinically erupted. Both maxillary and mandibular arches had reduced vertical bone height, loss of vestibular depth, and thin alveolar ridges (Fig. 2).

Panoramic radiographs were obtained when the child was 4 years. The radiograph showed a single deciduous lower left canine and few developing teeth in both maxillary and mandibular arches. The jaws appear to be underdeveloped, especially the maxilla. Several permanent teeth buds were missing (Fig. 3).

As a single mandibular canine was clinically present, we decided to fabricate a removable $\mathrm{Cu}$-sil denture in relation to the mandibular arch, and a complete denture for the maxillary arch. Diagnostic impressions were made using hydrocolloid material (alginate) with stock tray for mandible and an elastomeric material on a modified stock tray for maxilla (posterior surface of regular tray was shortened using a metallic disc and anterior flanges were bent) (Fig. 4).

Jaw relations were recorded and after verification, dentures were fabricated. Artificial teeth were modified to simulate deciduous teeth. Polished removable dentures were inserted and instructions were given to the parents regarding use and maintenance. The dentures significantly improved the esthetics of the child and also helped in mastication. Recall visits were scheduled to evaluate the compliance to the dentures and planned to reline or a new set of dentures to accommodate the growth of the child.

On the first recall visit on the next day of denture insertion, parents complained about excessive salivation. But, the child was

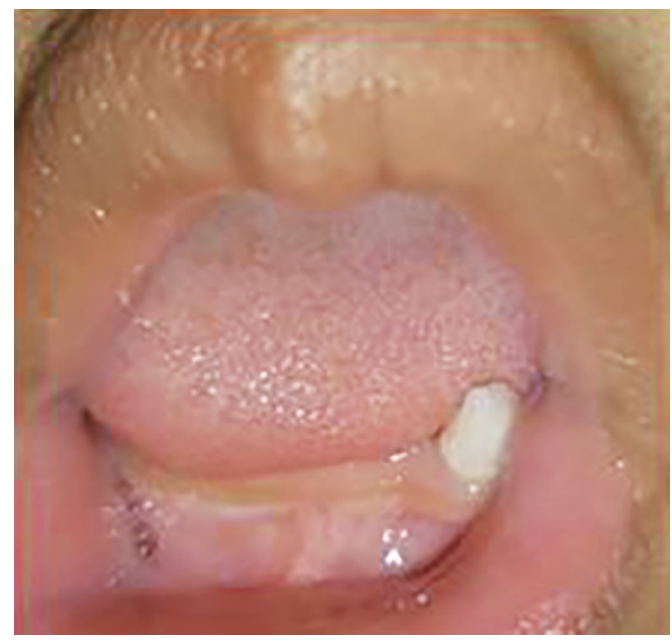

Fig. 2: Intraoral image of mandibular arch shows left deciduous canine 73

not ready to remove the upper denture. In the monthly review, the patient showed good compliance with the dentures (Fig. 5).

\section{Discussion}

Congenital hypothyroidism is a birth defect that can represent a pediatric emergency. Thyroid hormone is essential for normal growth and neurologic development, particularly in the first few years of life, and hypothyroidism during this period is a leading cause of preventable intellectual disability worldwide. Neonatal screening programs aim to detect disease in all apparently healthy newborns. Neonatal screening programs have revealed the fact that worldwide CHT incidence is approximately $1: 4,000 .^{4}$

Newborn screening (NBS) and thyroid therapy started within 2 weeks of age can normalize cognitive development. Thyroid function tests added to NBS programs beginning in the mid-1970s facilitated early detection and treatment of neonates with $\mathrm{CH}$. Several countries have implemented widespread NBS to identify and treat $\mathrm{CH}$ in newborns, but it is not yet universal in some countries. $^{8}$

Many hereditary disorders exhibit oral manifestations like alterations in the tooth number or morphology which can be detected by radiographs. Though genetic and metabolic disorders are generally diagnosed by pediatricians and endocrinologists, oral symptoms must be considered by dental professionals. Simple dental treatments can considerably improve the patients' condition.

In the present case, the child patient showed hypodontia. Prosthodontics rehabilitation was recommended treatment.

Rehabilitation of missing teeth by implants is usually limited to patients in whom the craniofacial growth is assumed to be complete. So, it is safer to wait until the growth period is complete. Few recommendations are given for the use of implants in the prosthodontic rehabilitation of children afflicted with hypo- or anodontia. To minimize orofacial impairment concerning nutrition, speech, and esthetic appearance, the first prosthetic rehabilitation should be performed before school enrolment. In patients with anodontia, prosthodontic rehabilitation should start by means of full dentures; implant placement and restoration by overdentures should be considered in the mandibular canine region at the age of 5-10 years and in the maxilla at 6 to 10 years. The use of implants in children 

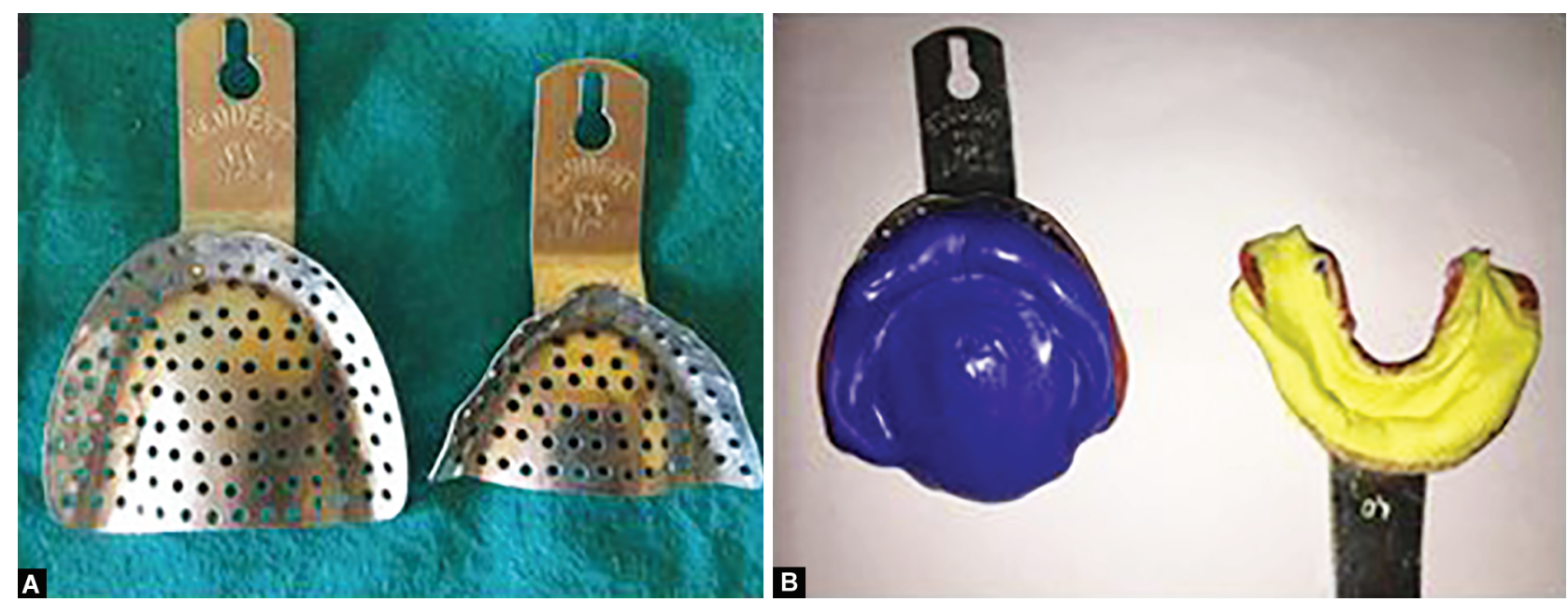

Figs 4A and B: (A) Modified maxillary stock tray (right) in comparison with a regular tray; (B) Impressions made using alginate and elastomeric impression materials
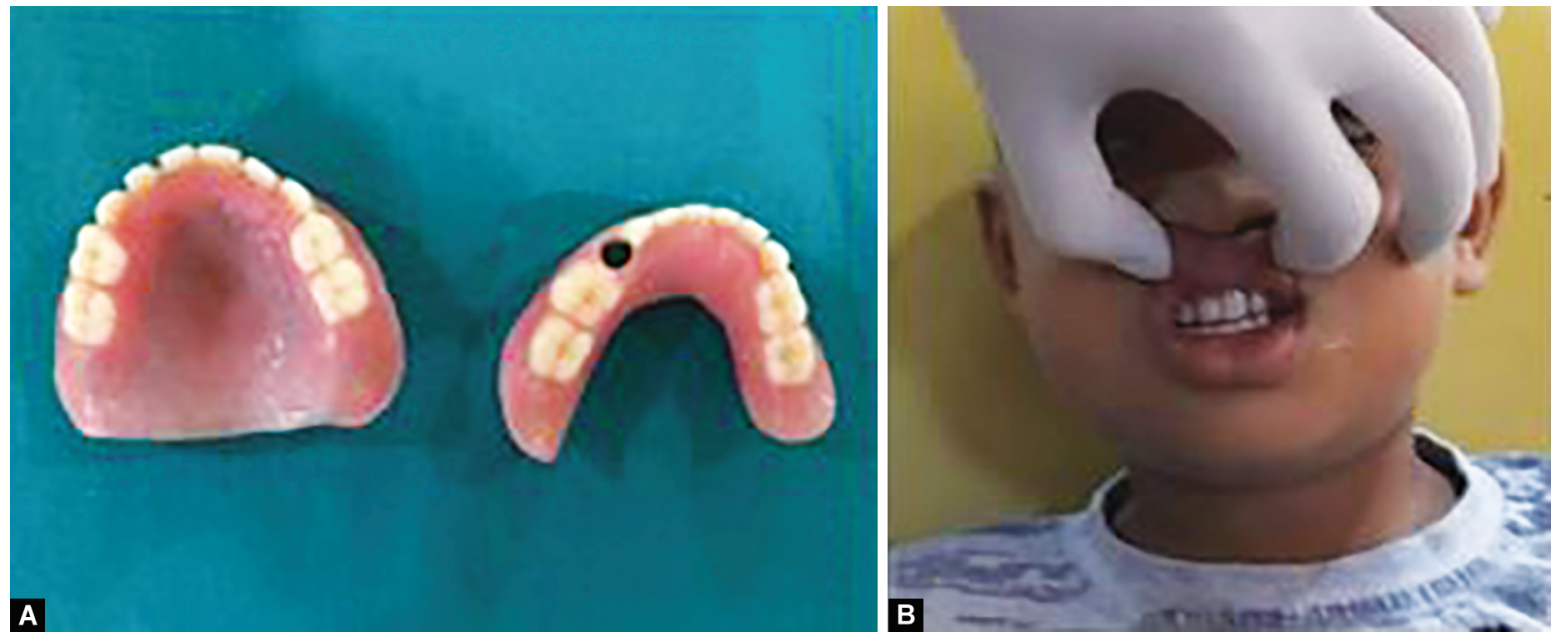

Figs $5 A$ and B: Extraoral view-front profile (during follow-up)

should be based on patient-specific requirements and that oral rehabilitation should be minimally invasive. ${ }^{9}$

Implant therapy was excluded as a treatment option due to the young age and the insufficient alveolar bone available. In children suffering from $\mathrm{CH}$, lack or absence of teeth with abnormal craniofacial vertical and sagittal skeletal relationships hampers the esthetics, masticatory ability, speech, and temporomandibular joint function.

The treatment plan of fabricating removable prostheses was planned considering the young age and underdeveloped arches. It also has an added advantage of early age intervention providing long-term psychosocial benefits. The maxillary arch was fully edentulous and treated with conventional denture design and the mandibular arch was treated with $\mathrm{Cu}$-sil denture. $\mathrm{Cu}$-sil denture is basically a complete denture with holes allowing the remaining natural teeth to emerge through the denture. ${ }^{10}$

Modifications of dental care must be considered while treating patients who have diseases of the thyroid gland. Patients with a history of thyroid diseases should be carefully evaluated to determine the level of medical management. Regular communication of the dentist with the endocrinologist is critical and regular clinical and laboratory investigations are important.
The endocrinologist must be aware of oral manifestations of the disease and dentists must be updated on thyroid control medications to help them to maintain the patient's oral health. ${ }^{11}$

\section{Conclusion}

Children affected with $\mathrm{CH}$ often present with a compromised dental status. The overall management needs a multidisciplinary approach.

Early detection of a $\mathrm{CH}$ can prevent the associated complications. Thus, NBS for thyroid function should be a part of the normal routine. Screening of all infants should be performed between 2 days and 4 days of birth. If this is not possible, testing should be performed before discharge or within 7 days of birth.

Pedodontists have the challenge to treat them at the earliest to reduce future complications and also delicately handle the psychosocial implications to the child patient. The children require monitoring throughout their lives, particularly more during early childhood.

\section{References}

1. Rastogi MVLS. Congenital hypothyroidism. Orphanet J Rare Dis 2010;5(1):17. DOI: 10.1186/1750-1172-5-17. 
2. Rodríguez ME, García MA, Flores IS. Congenital hypothyrodism and its oral manifestations. Rev Odontológica Mex 2014;18(2):132-137. DOI: 10.1016/S1870-199X(14)72063-4.

3. Kopel J. A global perspective on newborn congenital hypothyroidism screening. Proc (Bayl Univ Med Cent) 2019;33(1):137-139. DOI: 10.1080/08998280.2019.1668715.

4. Suma GN, Lakhanpal M, Dhillon M, et al. Orofacial manifestations of congenital hypothyroidism: Clinicoradiological case report. J Indian Acad Oral Med Radiol 2014;26(1):111-114. DOI: 10.4103/09721363.141876.

5. Young ER. The thyroid gland and the dental practitioner. J Can Dent Assoc 1989;55(11):903-907.

6. Loevy HT, Aduss H, Rosenthal IM. Tooth eruption and craniofacial development in congenital hypothyroidism: report of case. J Am Dent Assoc 1987;115(3):429-431. DOI: 10.14219/jada.archive.1987.0254.
7. Dudhia SB, Bhavin DB. Undetected hypothyroidism: A rare dental diagnosis. J Oral Maxillofac Pathol 2014;18(2):315. DOI: 10.4103/0973029X.140922.

8. Brown RS. Update of newborn screening and therapy for congenital hypothyroidism. Pediatrics 2006;117(6):2290-2303. DOI: 10.1542/ peds.2006-0915.

9. Schnabl D, Grunert I, Schmuth M, et al. Prosthetic rehabilitation of patients with hypohidrotic ectodermal dysplasia: A systematic review. J Oral Rehabil 2018;45(7):555-570. DOI: 10.1111/joor.12638.

10. Jain JK, Prabhu CR, Zahrane MA, et al. Cu-sil dentures - a novel approach to conserve few remaining teeth: Case reports. J Int Oral Health 2015;7(8):138-140.

11. Chandna S, Bathla M. Oral manifestations of thyroid disorders and its management. Indian J Endocrinol Metab 2011;15(6):113. DOI: $10.4103 / 2230-8210.83343$ 